\title{
Mediación tecnológica para desarrollar la competencia didáctica de futuros docentes de inglés como lengua extranajera
}

Technological mediation to develop the didactic competence in future English language teachers

Fecha de recepción: 28 de marzo de 2008 Fecha de aceptación: 14 de julio de 2008

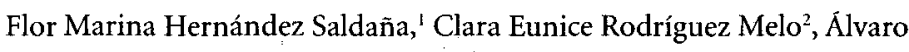
Hernán Quintero Polo ${ }^{3}$, Francisco Ramos Cuncanchún ${ }^{4}$

\section{Resumen}

Este artículo, de investigación científica y tecnológica, da cuenta de la tercera fase del proceso de investigación del proyecto Hacia un mejoramiento de mi quehacer como docente de Inglés: El software educativo un medio para solucionar algunos problemas metodológicos de inglés en docentes en formación. En las fases anteriores se había avanzado en la exploración y descripción de la situación problémica, diseñado la arquitectura de un software, el mapa de navegación, algunas interfases de las primeras tareas y realizado pre-pruebas del material, las cuales arrojaron la información que posibilitó continuar con la investigación (Hernández y Rodríguez 2002). El objetivo principal de la tercera fase fue re-diseñar el software acorde con las necesidades manifiestas en torno a la didáctica de los estudiantes de pre-grado de la Licenciatura en Educación Básica con Énfasis en Inglés (LEBEI) de la Universidad Distrital. Para la tercera fase del proyecto de investigación se concluyó que la intención era proponer una alternativa que sirviera como medio, no como fin en sí mismo, para el desarrollo de alguna competencia didáctica en los docentes de inglés en formación. De este modo, vimos que hacer una transición desde el método pre-experimental al descriptivo e interpretativo (Merriam, 1998) era lo más adecuado. Los resultados obtenidos fueron el software ElT (English Language Teaching), la plataforma DiT-on line y el uso de la tarea pedagógica Task para desarrollar la competencia didáctica.

\section{Palabras clave}

Software educativo, mediación tecnológica, formación de maestros, plataforma virtual, competencia didáctica.

1 Doctora en Ciencias Pedagógicas, ISEJV Habana, Cuba. mhernandezsf@yahoo.com

2 Magíster en Lingüistica Aplicada TEFL. Universidad Distrital Francisco José de Caldas. clareur@gmail.com

3 Magíster en Lingüística Aplicada TEFL. Universidad Distrital Francisco José de Caldas. quinteropolo@gmail.com

4 Doctor en Ciencias Pedagógicas, ISEJV Habana, Cuba. Larisateatro@gmail.com 


\section{Summary}

This scientific and technological article summarizes the third phase of the research Hacia un mejoramiento de mi quehacer como docente de inglés: El sofftware educativo un medio para solucionar algunos problemas metodológicos de inglés en docentes en formación. This phase was justified because of the previous ones, where some important aspects of the problem on exploration and description were raised; as well as the design and the architecture of the software, the navigation map and the design of some tasks. The piloting of the initial material gathered relevant information, which let the continuous process of the research go on. The main purpose of this third phase was to design educational software according to the students needs in relation to didactic competence of pre-service English language teachers from the Licenciatura en educación básica con énfasis en inglés (LEBEI) program at Distrital University. The third phase in its initial stage was conceived as a pre-experimental research (Larsen-Freeman, 1997). Later, it was stated that the intention was to propose a software application to be used as a means, not as an end, to develop the didactic competence of the pre-service English language teachers. That was how the research turned over from pre-experimental towards descriptive and interpretative research (Merriam, 1998) as the most appropriate research method. The results of the third phase were: The software ELT (English Language Teaching) the Dit on line platform and the application of tasks to develop the didactic competence.

\section{Key words}

Educational software, technological mediation, teacher education, Virtual platform, didactic competence.

\section{Introducción}

El grupo de investigación DIT (Didáctica del Inglés y. Tecnología) de la Universidad Distrital se ha propuesto ofrecer alternativas metodológicas que contribuyan a la adquisición de alguna competencia didáctica para la enseñanza del inglés como lengua extranjera, a través de entornos virtuales. En consonancia con esto, la tercera fase de la investigación propuso un material virtual que posibilitara complementar la formación inicial de profesores de inglés. En este artículo exponemos inicialmente algunos postulados teóricos resultantes de un rastreo bibliográfico sobre temáticas como: las competencias esenciales del profesor de inglés, las que hacen referencia al saber pedagógico dentro del cual se hace especial estudio de la didáctica, el enfoque basado en tareas, la tecnología como medio de enseñanza y el aprendizaje virtual (e-learning). En una segunda parte se describen las características de la propuesta del material tecnológico, allí se consideran aspectos pedagógicos, metodológicos y técnicos. Aparte del artículo se encuentra también el resumen de los principales hallazgos y de algunos componentes del software diseñado. Finalmente presentamos las conclusiones en donde intentamos responder la pregunta de investigación y proponemos unas implicaciones para la implementación del software.

Para comenzar, presentamos nuestro concepto de competencia, el cual expresamos así: "Competencia es la construcción social de lecciones significantes y útiles para el desempeño productivo en una situación educativa real, la cual se obtiene no solo por medio de instrucción, sino también, y en gran medida, por medio del aprendizaje por experiencia en situaciones educativas específicas" (Hernández y Quintero, 2005:22).

Las competencias profesionales las entendemos como un conjunto de conocimientos, destrezas, y aptitudes necesarias para ejercer una profesión, resolver problemas profesionales de forma autónoma y flexible y ser capaz de colaborar en el entorno profesional y en la organización del trabajo. Cada competencia es el producto de una combinación de recursos. El profesional utiliza un doble equipamiento para construir sus competencias: el primero incorporado 
a su persona: ser, saber, saber hacer, el segundo a su experiencia, medios y red de información (Bunk, citado por Coll, 1993).

En una mirada a los aspectos que conforman al profesional de la educación volcamos nuestra atención a la competencia pedagógica que asumimos (basados en Calderón, 2002) como una competencia macro conformada por otras competencias, que permite las relaciones entre ellas y que da como resultado una estructura base constituida por un dominio sobre una disciplina y sus condiciones de aprendizaje y de enseñanza. Estos dominios serán: idoneidad disciplinar y saber didáctico; dominio sobre el contexto de la educación (saber contextual y social); dominio ético (un saber ético, epistemológico y humano). Estos tres saberes específicos constituyen el saber pedagógico del docente. Finalmente, para nosotros, la competencia didáctica se relaciona con la habilidad de poner en relación armónica los elementos constitutivos del proceso docente en favor de los estudiantes en un contexto social determinado, que no se limita a su actuación en el salón de clase ni a la función meramente metodológica del profesor, sino que trasciende a todo el ámbito de su actuación como ser ético, como profesional de la educación.

En consecuencia de lo expresado hasta aquí, es posible concluir que el profesor de inglés debe ser equipado, entre otras competencias profesionales, de los componentes pedagógico y didáctico. Debe también desarrollar altos niveles de autonomía y asumir una posición crítica desde la pedagogía. El conjunto de los elementos mencionados propician las condiciones para que el profesor de lengua extranjera trabaje en la formación de los valores universales y los específicos del contexto socio-político de su país y es por ello que la didáctica necesita ser abordada no sólo en su función instrumental, sino como fuente de reflexión para el crecimiento profesional.

Para explicar la alternativa metodológica de la enseñanza a través de tareas, que es por la que hemos decido optar en nuestro software, acudimos a Breen (1987:23) quien define la tarea (task) como "cualquier esfuerzo estructurado de aprendizaje de una lengua que consta de un objetivo, un contenido, un procedimiento y una serie de resultados logrados por quien hace la tarea". La tarea es entonces asumida como una serie de planes de trabajo, formas de resolver un problema, hacer una simulación y/o tomar decisiones que tienen el propósito de facilitar el aprendizaje de lenguas. En consecuencia, nosotros vemos que el concepto de tarea tiene que ver con la actividad en donde la lengua extranjera es utilizada por el alumno con un propósito comunicativo para obtener un resultado (Willis, 1999).

Por lo anterior, el grupo de investigación asume el enfoque de tareas como medio para el proceso de aprendizaje-enseñanza y para fundamentar la propuesta metodológica porque nos ofrece un abanico de posibilidades para desarrollar no solo la competencia comunicativa sino también para la competencia didáctica del futuro profesor de inglés y mostrar que como formadores de formadores podemos volcar nuestra mirada hacia didácticas basadas en quien aprende.

Refiriéndonos al avance tecnológico y a las nuevas formas de enseñar y aprender en el mundo cambiante, asumimos que estas nos posibilitan incursionar en e-learning como una oportunidad para mediar el aprendizaje y utilizar la tecnología como alternativa didáctica del profesor de este siglo.

Las implicaciones pedagógicas que de su uso se derivan, las formas de construir conocimiento, las ventajas y desventajas del diseño de plataformas para este tipo de aprendizaje, el rol que deben asumir los docentes y especialmente los formadores de formadores responsables de que la educación y la instrucción estén acordes con las expectativas y necesidades de los variables órdenes sociales, son algunos de los cuestionamientos alrededor de los cuales el grupo reflexiona. 
El crecimiento de clases en línea puede ser un medio para generar mayores ingresos a los sistemas educativos y proporcionar menores costos a los beneficiarios. Indudablemente, el anterior ha sido el argumento más fuerte para ofertar software educativo, clases virtuales y cursos en red, pero también podemos afirmar que existen otras intencionalidades cuando se diseñan este tipo de cursos, una de ellas es el ofrecer oportunidades de reflexión, ejercitación y estudio sobre aspectos que necesiten profundización al terminar la instrucción formal presencial de algunas asignaturas, como es el caso de la didáctica del inglés en la LEBEI de la Universidad Distrital.

Para terminar esta discusión teórica, podemos afirmar que para propiciar el mejoramiento y desarrollo de su quehacer profesional, el futuro profesor de inglés deberá acercarse a unos saberes generales y específicos propios; esta condensación hay que desatarla para que se manifiesten a través de unos desempeños que materializan su competencia disciplinar, pedagógica, comunicacional y su capacidad investigativa; aquellos pueden mejorados a través de herramientas tecnológicas diseñadas para tal fin.

\section{Materiales y métodos}

Para el diseño de la propuesta se tuvieron en cuenta: las necesidades de los docentes de inglés en formación, las indicaciones emanadas por los expertos en la metodología de la enseñanza del inglés, la experiencia del grupo de investigación en el campo de la didáctica de la enseñanza del inglés y lo sugerido por los autores de la teoría sobre diseño de software y sistemas de gestión de aprendizaje en entorno red.

El enfoque pedagógico utilizado en la propuesta es la metodología de tareas (task). La tarea se refiere aquí a una actividad que realiza el estudiante en un ambiente virtual dentro o fuera del salón de clase con el propósito de alcanzar un logro o una meta. Esta propuesta se ve puesta en práctica en una plataforma virtual que más adelante se detallará.
Como principal producto de la investigación se tienen tres módulos que conforman el curso virtual ELT (English Language Teaching), al cual se accede a través de la página wEBDIT (http://didaytec.udistrital.edu.co) por medio del link DIT On-Line (http:// didaytec.udistrital.edu.co/classroom.html). Estos elementos se describen así:

ELT (English Language Teaching) es el nombre del software que se ha diseñado teniendo en cuenta la pregunta científica y las tareas presupuestadas para lograr el objetivo de la investigación, el cual es la elaboración de una propuesta de software para desarrollar la competencia didáctica del futuro profesor de lengua inglesa.

El software ELT está diseñado de modo que los temas pueden ser explorados separadamente en cualquier orden; los usuarios no necesitan realizar todas las actividades, solo aquellas que estén de acuerdo con sus intereses o necesidades. El software puede también ser utilizado en red como herramienta suplementaria en la clase de metodología de la enseñanza del inglés. Los módulos Methodology, Classroom Management, y Evaluation ofrecen en su orden 18, 16 y 6 tareas agrupadas en sub-temas. Así por ejemplo, en el módulo Methodology se encuentran tareas relacionadas con pensamiento crítico y desarrollo de destrezas psicomotoras del lenguaje. Las tareas están compuestas por los siguientes elementos estructurales:

Inicio: El nombre de la tarea se encuentra en la esquina superior izquierda; en la esquina superior derecha tiene el nombre del tema que trata. En la parte inferior se encuentra el menú de la tarea el cual contiene los demás elementos estructurales: HELP, AIM, INSTRUCTIONS, TUTOR CORNER, ASSESSMENT, Y GLOSARY.

Help: Este botón lleva a un tutorial sobre el uso y la descripción de cada botón de la tarea.

Aim: Los objetivos de la tarea 

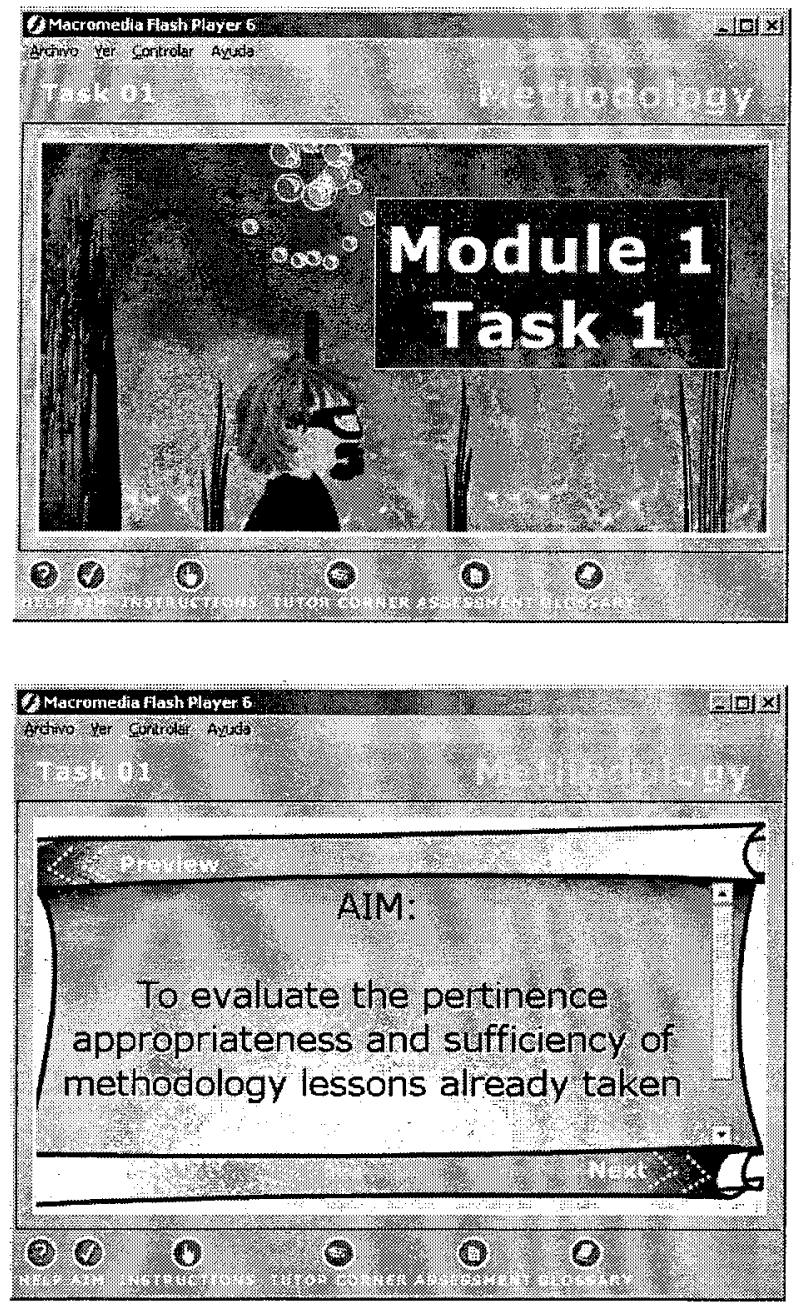

Instructions: Las instrucciones, y procedimientos para realizar la tarea.

Tutor Corner: Hace referencia a lecturas y ayudas, como videos, complementarios a las tareas y las instrucciones.

Assessment: Permite por medio de preguntas que el estudiante reflexione sobre lo realizado.

Glossary: Ofrece definiciones de términos utilizados en las tareas.

ELT software sigue una metodología con posición constructivista en donde las tareas y actividades propuestas contribuyen a la auto-construcción de conocimiento social, humano y cultural del futuro profesor de inglés. Las tareas conducen a los futuros

CENTRO DE INVESTIGACIONES Y DESARROLLO CIENTÍFICO
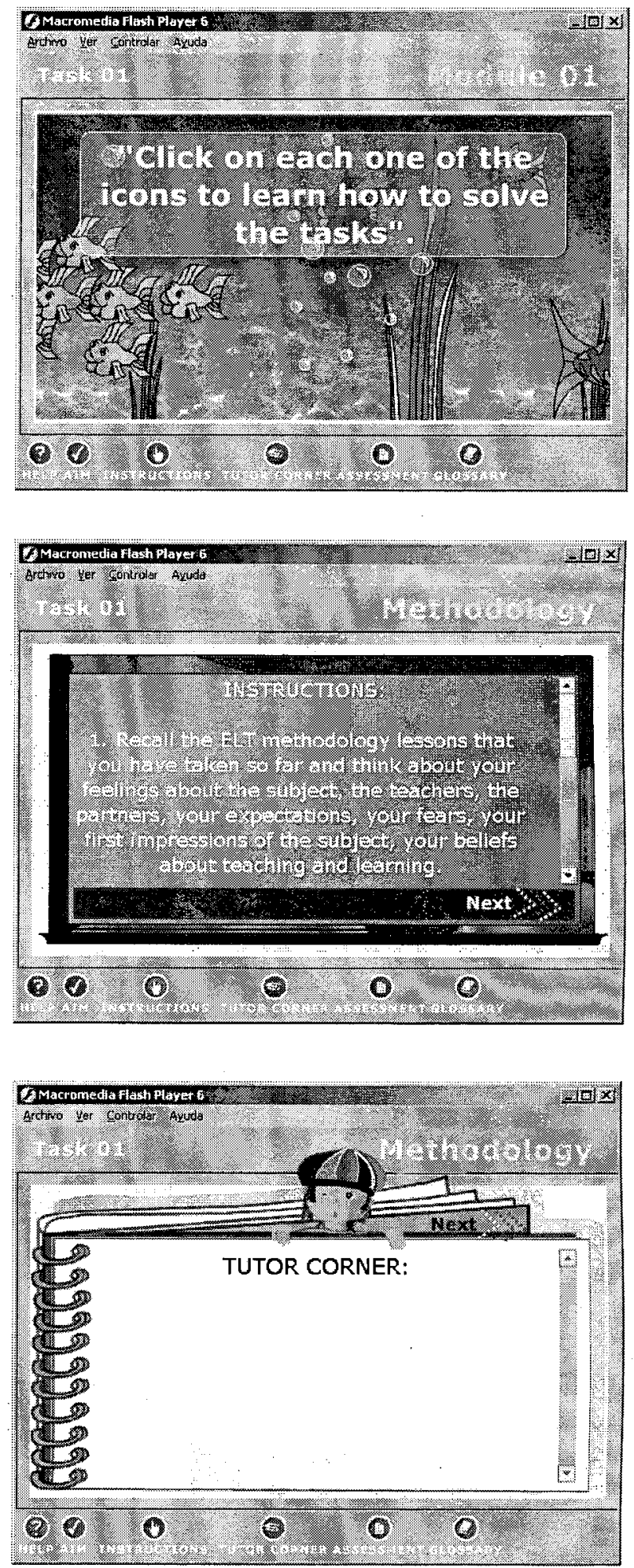

profesores de inglés hacia la reflexión sobre su propia experiencia y su progreso en la competencia.. Los usuarios no son dirigidos hacia un patrón rígido de cómo enseñar, ellos exploran diferentes opciones y luego escogen la alternativa más adecuada de 


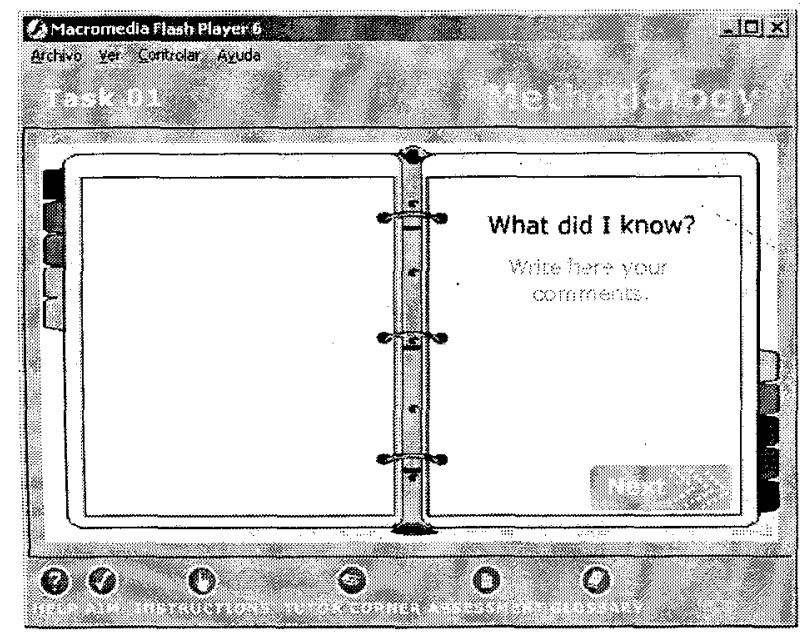

acuerdo a su contexto. La navegación es heurística, el usuario tiene acceso a cualquier tarea o parte de una tarea. Para su desarrollo se tuvo como principal fuente de alimentación el guión descriptivo y la arquitectura del software realizada por el grupo de investigación.

La evaluación en el software consiste en una serie de consideraciones sobre la forma como se desarrollan las tareas, que llevan al futuro profesor de inglés a la valoración de su propio desempeño, a la constatación de sus saberes, a la toma de decisiones y a evidenciar su progreso individual de aprendizaje.

webdit es el nombre de la página web del grupo de investigación http://www.didaytec.udistrital.edu. co. Esta página se instrumenta como la forma para dar presentación y posicionamiento internacional al grupo de investigación. Ella ofrece su imagen, la misión y la visión del mismo, permite el acceso a la información sobre publicaciones, eventos realizados y programados, además ilustra sobre los productos y servicios del grupo.

La página del grupo coṇsta de los siguientes ítems:

Inicio: Es la pagina que recibe al visitante, en ella encontrará información de la Universidad Distrital, Colciencias, Centro de Investigaciones y Desarrollo Científico de la Universidad Distrital, una pizarra de información general, y un espacio para la informa-

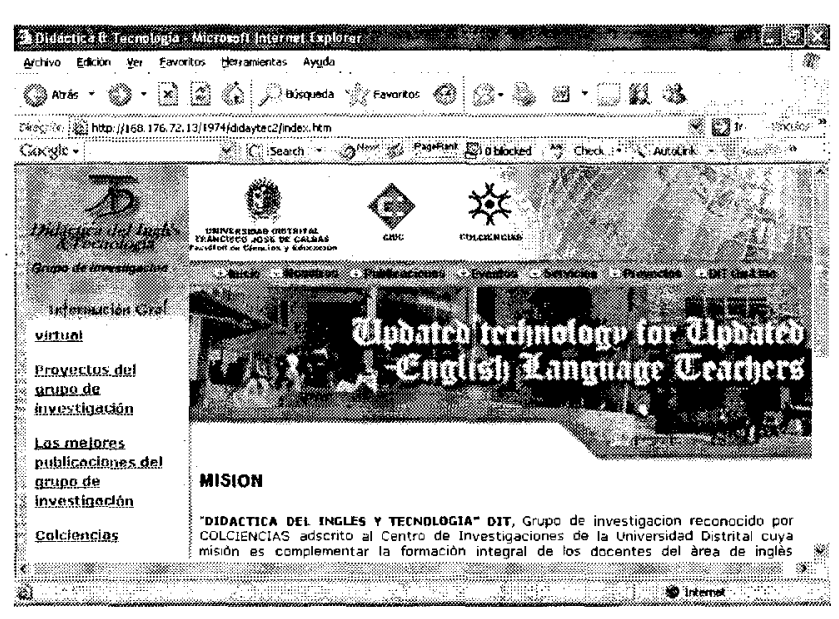

ción importante del momento. En la parte superior tiene un menú que presenta los demás links: Nosotros, Publicaciones, Eventos, Servicios, Proyectos, y DIT on-line

Nosotros: Describe a cada uno de los integrantes e investigadores que conforman el grupo DIT y posibilita la comunicación electrónica con cada uno de ellos. Responde a dos interrogantes: ¿Quiénes Somos? y ¿A qué redes pertenecemos?

Publicaciones: El grupo de investigación se centra en los productos y con ellos se hace visible a la comunidad. En esta sección se encontraran las publicaciones del grupo.

Eventos: Es el icono que ilustra y actualiza sobre la participación que hace el grupo en diferentes escenarios académicos a nivel nacional e internacional.

Servicios: Es la parte de la página Web que informa a la comunidad acerca de los campos de acción en que se desempeña el grupo.

Proyectos: Se refiere a las investigaciones concluidas, en curso y a las que se proyectan.

dit on-line: Es la puerta de entrada a la plataforma virtual a la que se accede por medio de la webdit (http://www.didaytec.udistrital.edu.co) en el botón dit on-line. Se despliega una interfaz que pide un 


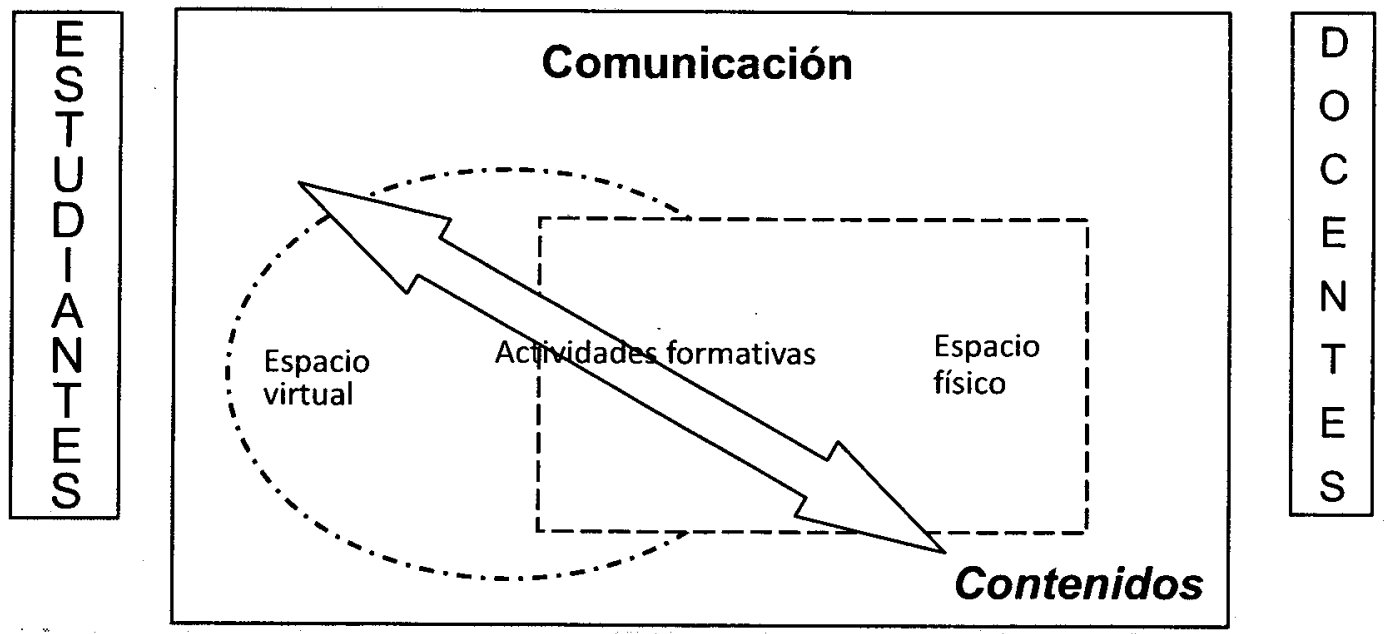

login y una contraseña, que son suministradas por el administrador. Se puede ingresar en calidad de docente o en calidad de estudiante de alguno de los cursos.

La plataforma permite que los docentes creen sus cursos y sistemas de gestión de aprendizaje en entorno web. dit on-line es el instrumento que opera el software elt, el curso virtual y que además (entre sus otras bondades) posibilita la creación de cursos virtuales que sigan un modelo híbrido que se explica a continuación:

Modelo Híbrido se asume aquí como una forma de enseñanza y aprendizaje que integra la virtualidad con la presencialidad, tal y como lo describe Alpiste, F. (2004) en su disertación de tesis doctoral de la Universidad de Cataluña Modelo de formación híbrido para el diseño, producción e integración de contenidos formativos y entornos virtuales de aprendizaje y de la cual presentamos la anterior figura con el objeto de explicar gráficamente el marco propuesto.

El espacio en el cual la formación tiene lugar se encuentra constituido por dos planos, uno físico y otro virtual, ambos igual de reales aunque con sus propias formas de funcionamiento. Las actividades formativas se organizan considerando simultáneamente las aulas (espacio físico) y la red (espacio virtual) y consisten en procesos de comunicación que aplican con múltiples estrategias los diversos tipos de contenidos. La comunicación puede ser sincrónica o asincrónica.

DIT on-line ofrece una interacción con los contenidos web que incluyen: videos de clases reales, foros, chat, apoyo y contacto con tutores. Las principales características de DIT on line son las siguientes: Core (foros, chat, documentos de descarga, notas, agenda), collaboration (sesiones de grupo, digitalización de audio y video, interactividad, horarios opcionales).

DIT on-line tiene los siguientes elementos: Administrador de la plataforma (crea, modifica, elimina cursos, docentes o estudiantes), el profesor (crea, modifica o elimina contenido de sus cursos, puede publicar documentos para compartirlos con los alumnos, ver los trabajos que éstos le envían, ver la lista de los estudiantes, conversar con ellos, digitar notas, crear foros para la discusión o tener datos compartidos con los estudiantes en una agenda), el alumno (ve el contenido del curso que el docente crea, envía trabajos, ve las notas y el promedio, conversa con el docente del curso y sus compañeros, participa en foros de discusión creados por el docente y visualiza los datos de la agenda del curso).

La plataforma utilizada para el modelamiento es UML (Lenguaje de Modelamiento Unificado) y luego de la que se utilizó el RuP (Proceso Unificado de Desarrollo de Software). Los requerimientos mínimos para correr el programa son: Procesador Pentium 
o AMD de $500 \mathrm{MHz}$ o superior, $128 \mathrm{Mb}$ de memoria RAM o superior, tarjeta de video compatible con VGA o superior, monitor a color y dispositivo de conexión a Internet.

\section{Resultados y discusión}

Los resultados resumidos en este apartado deben entenderse como una pre-prueba del aplicativo debido a que los encuestados exploraron una versión previa a la versión estable del software ELT. El aplicativo tenía activados los módulos uno y dos. Estos módulos tenían a su vez activadas unas tareas (tasks).

El procedimiento para una aproximación a las percepciones de unos usuarios acerca del software fue inicialmente convocar a un grupo de futuros docentes de inglés de sexto, séptimo y octavo semestres del proyecto curricular de Licenciatura en Educación Básica con Énfasis en Inglés de la Universidad Distrital Francisco José de Caldas. A la convocatoria respondieron 28 docentes en formación. Ellos asistieron a una sala de informática para explorar el modulo uno, que se encontraba en un CD-Rom. Al tiempo que exploraban el módulo, se les pidió que contestaran un cuestionario. No todos respondieron la totalidad de las preguntas de la encuesta, lo que hizo que no hubiera uniformidad en el número de personas que contestaron.

El software tenía activado únicamente las tareas. Otros elementos como las conexiones con la página web del grupo y el foro del tutor (speaking corner) no estaban activados lo que ocasionó que aspectos como navegación en la red no se pudieran realizar.

Los encuestados evaluaron los siguientes aspectos del módulo uno:

Contenidos: Aquí se buscaba averiguar hasta dónde los estudiantes podían establecer con claridad la información y hasta dónde estos contenidos se ajustaban a ellos como usuarios.
Texto: Aquí se intentaba averiguar si los estudiantes podían entender con claridad las instrucciones escritas, si estas estaban estructuradas de manera apropiada y si las ideas expresadas en ellos eran claras.

Imágenes y animaciones: Aquí se buscaba determinar el grado de relación entre las imágenes y el texto, su utilidad en términos de comunicación y su calidad estética y expresiva.

Enlaces: Aquí se quería establecer el nivel de eficiencia de los enlaces en la utilización del aplicativo.

Interfaz: Aquí se buscaba determinar la adecuación y la pertinencia de los contenidos presentados.

Color: Aquí se intentaba determinar cómo contribuye el color en la legibilidad de la presentación y hasta dónde aporta a la información.

Controles: Aquí se buscaba establecer la forma como los estudiantes podían hacer uso de este recurso para el desarrollo de las diferentes actividades.

De acuerdo con el análisis general de los resultados obtenidos en la muestra, se puede establecer que el módulo uno, correspondiente a metodología, tiene la mayor calificación en los aspectos de calidad, legibilidad de la animación, la utilización del color como aporte a la legibilidad y el manejo de las imágenes con relación al texto para no interferir con este. Otros aspectos que se destacaron en sentido positivo en la evaluación corresponden a la estética y expresividad de la animación sobre la cual podrían implementarse algunas alternativas para enriquecerla.

En vista de los resultados anteriores, vimos que era necesario pedir a otro grupo de futuros docentes de inglés que nos brindaran sus apreciaciones acerca de aspectos más puntuales del software ELT. A continuación se describen los resultados de un segundo grupo de encuestas acerca de la muestra del software ELT: tareas, módulos uno y dos. 
Para esta segunda encuesta, convocamos a un grupo de 49 estudiantes de sexto, séptimo y octavo semestres del proyecto curricular de Licenciatura en Educación Básica con Énfasis en Inglés de la Universidad Distrital Francisco José de Caldas. En una sala de informática de la Universidad Distrital se llevó a cabo una exploración de unas tareas (tasks) contenidas en una versión preliminar del software ELT, particularmente de los módulos uno y dos. De estos dos módulos, los estudiantes seleccionaron cinco tareas libremente. Al igual que en la descripción de resultados anterior, el aplicativo tenía activado únicamente las tareas. Otros elementos como las conexiones con la página web y el la esquina del tutor (speaking corner) no estaban activados. Los encuestados evaluaron los siguientes aspectos de las tareas:

Aims (objetivos): Aquí se buscaba averiguar la medida en la que los objetivos eran claros, apropiados, interesantes y motivadores.

Instructions (instrucciones): Aquí se intentaba averiguar si las instrucciones eran claras, suficientes y que mostraran una secuencia.

Activities (actividades): Aquí se buscaba determinar si las actividades eran apropiadas para los objetivos de la tarea, si promovían reflexión, si se podían resolver sin necesidad de una secuencia y si promovían aprendizaje individual y colectivo.

Assessment (evaluación): Aquí se quería observar la medida en que las tareas contenían alternativas de evaluación que promovieran la reflexión, que fueran consistentes con los objetivos y con las actividades y que promovieran la auto evaluación del desempeño de los estudiantes.

Content (contenidos): Aquí se buscaba determinar si el contenido involucraba los intereses de los estudiantes, si era relevante para el desarrollo profesional, si daba oportunidades para el aprendizaje autónomo y si era diferente de una clase presencial.
Interaction (interacción): Aquí se intentaba determinar si era fácil completar las tareas, si los íconos eran claros para ser usados, si el speaking corner era fácil de leer y si las actividades mostraban algún grado de dificultad.

Se inició el análisis de las respuestas haciendo énfasis en las tendencias hacia los aspectos positivos (valores 4 a 5 en las encuestas). De la misma manera, los aspectos negativos (valores 1 a 3 ) se revisaron a fin de hacer los cambios pertinentes en las tareas evaluadas. Nos dimos cuenta de el contraste de estas dos tendencias en donde la mayoría de estudiantes resaltan el ítem de contenido como el mejor y el ítem de interacción como el más débil.

Como una forma de complementar las apreciaciones resultantes de las encuestas anteriores, creímos que era importante tener una visión acerca del aspecto de evaluación ya que este reflejaba una de las debilidades del aplicativo. Las siguientes preguntas estuvieron contenidas en la entrevista acerca de las percepciones sobre evaluación:

1. ¿Qué concepción tiene acerca de evaluación y qué busca a través de ella?

2. ¿Cómo ha vivido la evaluación a lo largo de su proceso de aprendizaje?

3. ¿Cómo ha sido evaluado y cómo ha dado respuesta a dicho proceso?

4. ¿Qué papel ha cumplido la evaluación e su proceso formativo?

5. ¿Qué formas de evaluación utiliza en su práctica docente?

6. ¿Qué acciones toma basado en los resultados que este tipo de evaluación arroja?

7. ¿Qué papel ha cumplido la auto evaluación en su desarrollo profesional? 
Esta entrevista surgió como una necesidad de expandir las apreciaciones de nueve encuestados quienes habían tenido anteriormente la oportunidad de explorar unas tareas en el software ELT. En la siguiente tabla se resumen las percepciones por cada aspecto explorado en la entrevista.

En una mirada detallada de las respuestas, podemos destacar que el aspecto de evaluación se percibe como una actividad que implica un proceso sistemático de recolección de datos con el fin de interpretarlos y luego documentar unas acciones o decisiones acerca de actividades educacionales. Vemos además que las experiencias con la evaluación están determinadas por los contextos y niveles educativos de los entrevistados. Esto hace que se pueda apreciar una transición de un enfoque cuantitativo a un enfoque cualitativo relacionado con evaluar. Sobre esto se destaca también el componente reflexivo presente en los procesos evaluativos de los entrevistados. Siguiendo en la misma línea, la evaluación toma varias formas para los entrevistados; sin embargo, existe una tendencia a mantener las formas sumativas de evaluación antes que implementar transformaciones en las prácticas evaluativas debido a factores externos. Finalmente, dentro de las acciones mencionadas por los entrevistados se aprecia la necesidad de incluir la investigación y la autorreflexión como componentes del proceso evaluativo en contextos educacionales.

Teniendo en cuenta los resultados de esta pre-prueba del software educativo ELT, el grupo de investigación procedió a la revisión, refuerzo y corrección de los aspectos resaltados en la lectura de las encuestas. A continuación describimos la propuesta de la mediación tecnológica en nuestro proyecto investigativo.

\section{Conclusiones}

La tercera etapa de nuestra investigación constituye una transición para nosotros los investigadores. Esta transición tiene que ver con un cambio de perspectiva desde donde vemos ahora el software educativo.
En etapas anteriores de este proyecto, concebíamos el software educativo como un fin hacia lo cual todo esfuerzo tanto pedagógico como investigativo debía dirigirse. Sin embargo, en esta etapa hemos aprendido que lo que se mostraba como más relevante no era el recurso tecnológico en sí mismo, sino lo que pudiera explorarse a través de él. Es así entonces como nuestra perspectiva varía hacia ver el software educativo como un medio para contribuir al desarrollo de competencias profesionales de nuestros futuros docentes de inglés. De este mismo modo, vimos que esta perspectiva nos permitió ser consistentes con la intención de responder el interrogante investigativo que propusimos en un comienzo.

Con el fin de dar respuesta a la pregunta de investigación ¿Cómo contribuir a mejorar la competencia didáctica del futuro profesor de inglés? Encontramos que nuestra alternativa virtual constituye una mediación entre un componente teórico y uno práctico en el proceso de formación de futuros docentes de inglés. Esta mediación, la cual inicialmente contemplaba únicamente el diseño y programación de un software educativo, tomó la forma de unos recursos estables a saber, un software llamado ELT, una página web llamada wEBDIT y una plataforma virtual llamada Web DIT on-line.

Estos recursos contienen herramientas y actividades dirigidas a fomentar la autonomía, la autorreflexión, y en general el auto aprendizaje como un complemento a las sesiones presenciales a las que los futuros docentes de inglés deben asistir. En consecuencia, creemos que la alternativa virtual que presentamos en esta investigación es una contribución al desarrollo de la competencia metodológica de nuestros futuros docentes de inglés.

Esta alternativa es una innovación porque es la primera de su género en el contexto colombiano. A pesar de la proliferación de cursos virtuales, aún no se había ofrecido a la comunidad académica un aplicativo diseñado para mejorar las competencias profesionales de los futuros docentes de inglés, 
basado en videos de clases reales. Además, es una innovación porque sugiere la combinación de la clase presencial con la clase virtual lo cual permite a los usuarios mayores espacios para la auto reflexión, la autonomía y la aplicación de los principios de la pedagogía crítica.

El saber pedagógico del futuro profesor de inglés se refleja en la forma como el docente aborda su labor en la práctica y durante las intervenciones en los espacios de aula. Es por ello que los videos recolectados de clase de profesionales del área de inglés permitieron hacer explícitos los fundamentos teóricos que subyacen algunas veces inconscientes.

Como una forma de recoger las impresiones de un grupo de cuarenta futuros docentes de inglés al tiempo que ellos realizaban una exploración de una versión previa a la versión estable del programa (pre-prueba), llevamos a cabo una indagación por medio de encuestas. Ellas tuvieron por objeto averiguar cuáles eran las apreciaciones de los encuestados acerca de la dimensión técnica e instrumental del software y también la dimensión conceptual de los contenidos. Pudimos averiguar que dos tendencias, una positiva y otra negativa, reflejaron los aspectos a reforzar y los aspectos a corregir. Podemos destacar el aspecto de evaluación como uno de los cuales debe ser reforzado en el aplicativo. Sobre esto, realizamos unas entrevistas, como una forma de expandir la información de los encuestados, acerca de sus apreciaciones sobre el componente evaluativo del software.

Finalmente, otro aspecto a destacar en esta sección de conclusiones es el del impacto de esta investigación en el currículo de la Licenciatura en Educación Básica con Énfasis en Inglés de la Universidad Distrital. Este impacto tiene que ver con la introducción de un componente tecnológico en la formación de docentes de inglés y además la presentación de una plataforma virtual en donde tanto formadores de docentes como docentes en formación puedan interactuar entre sí y con otros usuarios externos a la Universidad Distrital en torno a temas pedagógicos e investigativos. Esto está estrechamente ligado a la promoción del aprendizaje autónomo, lo cual está implícito en nuestra investigación. Dentro de la concepción del impacto de esta investigación también vemos que internamente el grupo de investigación fue objeto de transformaciones en cuanto a sus miembros y las experiencias investigativas. El grupo, inicialmente conformado por docentes, vio la necesidad de apoyarse en un equipo tecnológico que diera forma a las propuestas pedagógicas en unos recursos virtuales. De igual modo se generaron oportunidades tanto para aprendices de investigación (pasantes) como para docentes investigadores de nutrirse de y hacer aportes para el proyecto de investigación.

\section{Implicaciones para práctica pedagógica futura}

Dentro de los resultados esperados se aspira a familiarizar a los futuros maestros de inglés con el uso de la herramienta tecnológica para desarrollar un aprendizaje autónomo que sirva tanto para su mejoramiento como docentes, como para usar y vincular el computador al aula de clase de inglés. El software, producto de la investigación, puede ser utilizado en contextos académicos y universidades que presenten problemas parecidos a los detectados en esta investigación a nivel nacional e internacional. Además, se espera que se fortalezca y se consolide el semillero de jóvenes docentes investigadores en la didáctica del inglés y la tecnología por medio de propuestas de trabajos de grado.

\section{Bibliografía}

ALPISTE, F. (2004). Modelo de formación híbrido para el diseño, producción e integración de contenidos formativos $y$ entornos virtuales de aprendizaje, Universidad de Cataluña.

BREEN, M. (1987). Learner Contribution to Task Design. Oxford, Pergamon Press. 
COLL, C. et. al. (1993). El Constructivismo en el aula. Editorial Graó. Barcelona,

CALDERON, D. (2002). Utopías sobre el nuevo maestro que requiere Colombia. Memorias Congreso Nacional de Maestros Formar. Medellín.

HERNÁNDEZ y QUINTERO. (2005). Un espacio virtual para la metodología de la enseñanza del inglés, Fondo de Publicaciones de la Universidad Distrital. Bogotá.

HERNÁNDEZ, M. y RODRÍGUEZ, C. (2002). Alternativa de un mundo virtual para mejorar su enseñanza de inglés. Fondo de Publicaciones de la Universidad Distrital. Bogotá.
MERRIAN. (1998). Qualitative Research And Case Study Applications In Education. San Francisco, Jossey Bass.

LARSEN-FREEMAN, D. (1997). An Introduction To Second Language Acquisition Research. Londres, Longman.

LARSEN-FREEMAN, D. y LONG, M. (1994). Second Language Acquisition: an introduction to second language acquisition Research. Londres, Longman.

WILLIS,J. (1999). A Framework For Task Based Learning, Malaysia, Longman. 